UDC: $517.518 .1 / .2$

43 (LXIX) 2 (2019), 31-52

DOI: $10.37560 /$ matbil $2190031 \mathrm{k}$

\title{
NEW TRAPEZIUM INEQUALITIES FOR GENERALIZED INTEGRAL OPERATORS PERTAINING $m$-CONVEX FUNCTIONS AND THEIR APPLICATIONS
}

\author{
ARTION KASHURI
}

\begin{abstract}
The author discovered an identity for a generalized integral operator via differentiable function. By using this integral equation, we derive some new bounds on Hermite-Hadamard type integral inequalities for differentiable functions that are in absolute value at certain powers $m$-convex. By taking suitable choices of function, some interesting results are obtained. At the end, some applications of presented results to special means and new error estimates for the trapezium formula have been analyzed. The ideas and techniques of this paper may stimulate further research in the field of integral inequalities.
\end{abstract}

\section{INTRODUCTION}

The following inequality, named Hermite-Hadamard inequality, is one of the most famous inequalities in the literature for convex functions.

Theorem 1.1. Let $f: I \subseteq \mathbb{R} \longrightarrow \mathbb{R}$ be a convex function and $r_{1}, r_{2} \in I$ with $r_{1}<r_{2}$. Then the following inequality holds:

$$
f\left(\frac{r_{1}+r_{2}}{2}\right) \leq \frac{1}{r_{2}-r_{1}} \int_{r_{1}}^{r_{2}} f(x) d x \leq \frac{f\left(r_{1}\right)+f\left(r_{2}\right)}{2} .
$$

This inequality (1.1) is also known as trapezium inequality.

The trapezium inequality has remained an area of great interest due to its wide applications in the field of mathematical analysis. Authors of recent decades have studied (1.1) in the premises of newly invented definitions due to motivation of convex function. Interested readers see the references [1][19, 21]-24.

The aim of this paper is to establish trapezium type generalized integral

2010 Mathematics Subject Classification. Primary: 26A51; Secondary: 26A33, 26D07, 26D10, 26D15.

Key words and phrases. Hermite-Hadamard inequality, Hölder's inequality, power mean inequality, $m$-convex, general fractional integrals. 
inequalities for $m$-convex functions. Interestingly, the special cases of presented results, are fractional integral inequalities. Therefore, it is important to summarize the study of fractional integrals.

Let us recall some special functions and evoke some basic definitions as follows:

Definition 1.2. [12] A function $f:\left[0, r_{2}\right] \longrightarrow \mathbb{R}$ is called $m$-convex with $m \in[0,1]$, if for any $x, y \in\left[0, r_{2}\right]$ and $t \in[0,1]$, we have

$$
f(t x+m(1-t) y) \leq t f(x)+m(1-t) f(y) .
$$

Definition 1.3. [16] Let $f \in L\left[r_{1}, r_{2}\right]$. Then $k$-fractional integrals of order $\alpha, k>0$ with $r_{1} \geq 0$ are defined by

$$
I_{r_{1}^{+}}^{\alpha, k} f(x)=\frac{1}{k \Gamma_{k}(\alpha)} \int_{r_{1}}^{x}(x-t)^{\frac{\alpha}{k}-1} f(t) d t, \quad x>r_{1}
$$

and

$$
I_{r_{2}^{-}}^{\alpha, k} f(x)=\frac{1}{k \Gamma_{k}(\alpha)} \int_{x}^{r_{2}}(t-x)^{\frac{\alpha}{k}-1} f(t) d t, \quad r_{2}>x .
$$

For $k=1, k$-fractional integrals give Riemann-Liouville integrals. For $\alpha=$ $k=1, k$-fractional integrals give classical integrals.

Also, let recall the function $\varphi:[0,+\infty) \longrightarrow[0,+\infty)$ satisfying the following conditions:

$$
\begin{aligned}
& \int_{0}^{1} \frac{\varphi(t)}{t} d t<+\infty, \\
& \frac{1}{A} \leq \frac{\varphi(s)}{\varphi(r)} \leq A \text { for } \frac{1}{2} \leq \frac{s}{r} \leq 2, \\
& \frac{\varphi(r)}{r^{2}} \leq B \frac{\varphi(s)}{s^{2}} \text { for } s \leq r, \\
& \left|\frac{\varphi(r)}{r^{2}}-\frac{\varphi(s)}{s^{2}}\right| \leq C|r-s| \frac{\varphi(r)}{r^{2}} \text { for } \frac{1}{2} \leq \frac{s}{r} \leq 2,
\end{aligned}
$$

where $A, B, C>0$ are independent of $r, s>0$. If $\varphi(r) r^{\alpha}$ is increasing for some $\alpha \geq 0$ and $\frac{\varphi(r)}{r^{\beta}}$ is decreasing for some $\beta \geq 0$, then $\varphi$ satisfies 1.3 (1.6), see [20]. Therefore, the left-sided and right-sided generalized integral operators are defined as follows:

$$
\begin{aligned}
r_{1}^{+} I_{\varphi} f(x) & =\int_{r_{1}}^{x} \frac{\varphi(x-t)}{x-t} f(t) d t, \quad x>r_{1}, \\
r_{2}^{-} I_{\varphi} f(x) & =\int_{x}^{r_{2}} \frac{\varphi(t-x)}{t-x} f(t) d t, \quad x<r_{2} .
\end{aligned}
$$


The most important feature of generalized integrals is that; they produce Riemann-Liouville fractional integrals, $k$-Riemann-Liouville fractional integrals, Katugampola fractional integrals, conformable fractional integrals, Hadamard fractional integrals, etc., see [19].

Motivated by the above literatures, the main objective of this paper is to discover in Section 2, an identity for a generalized integral operator via differentiable function. By using the established identity as an auxiliary result, some new estimates on Hermite-Hadamard type integral inequalities for differentiable functions that are in absolute value at certain powers $m$-convex are obtained. It is pointed out that some new fractional integral inequalities have been deduced by taking various suitable choices of function. In Section 3, some applications to special means and new error estimates for the trapezium formula are given. The ideas and techniques of this paper may stimulate further research in the field of integral inequalities.

\section{MAin RESUlts}

Throughout this study, let $P=\left[m r_{1}, r_{2}\right]$ with $r_{1}<r_{2}$ and $m \in(0,1]$. Also for brevity, we define

$$
\Lambda(t):=\int_{0}^{t} \frac{\varphi\left(\frac{\left(r_{2}-m r_{1}\right) u}{4}\right)}{u} d u<\infty, \quad \forall t \in[0,1] .
$$

For establishing some new results regarding general fractional integrals we need to prove the following lemma.

Lemma 2.1. Let $f: P \longrightarrow \mathbb{R}$ be a differentiable mapping on $\left(m r_{1}, r_{2}\right)$. If $f^{\prime} \in L(P)$, then the following identity for generalized fractional integrals hold:

$$
\begin{gathered}
\frac{1}{4}\left[f\left(\frac{3 m r_{1}+r_{2}}{4}\right)+f\left(\frac{m r_{1}+r_{2}}{2}\right)+f\left(\frac{m r_{1}+3 r_{2}}{4}\right)+f\left(r_{2}\right)\right] \\
-\frac{1}{4 \Lambda(1)}\left[\left(\frac{3 m r_{1}+r_{2}}{4}\right)-I_{\varphi} f\left(m r_{1}\right)+{ }_{\left(\frac{m r_{1}+r_{2}}{2}\right)}-I_{\varphi} f\left(\frac{3 m r_{1}+r_{2}}{4}\right)\right. \\
\left.+{ }_{\left(\frac{m r_{1}+3 r_{2}}{4}\right)}{ }^{-} I_{\varphi} f\left(\frac{m r_{1}+r_{2}}{2}\right)+{ }_{r_{2}^{-}} I_{\varphi} f\left(\frac{m r_{1}+3 r_{2}}{4}\right)\right] \\
=\frac{1}{4 \Lambda(1)} \int_{m r_{1}}^{r_{2}} p_{\Lambda}(t) f^{\prime}(t) d t
\end{gathered}
$$


where

$$
p_{\Lambda}(t):= \begin{cases}\Lambda\left(\frac{4\left(t-m r_{1}\right)}{r_{2}-m r_{1}}\right), & t \in\left[m r_{1}, \frac{3 m r_{1}+r_{2}}{4}\right) ; \\ \Lambda\left(\frac{4\left(t-\frac{3 m r_{1}+r_{2}}{4}\right)}{r_{2}-m r_{1}}\right), & t \in\left[\frac{3 m r_{1}+r_{2}}{4}, \frac{m r_{1}+r_{2}}{2}\right) ; \\ \Lambda\left(\frac{4\left(t-\frac{m r_{1}+r_{2}}{2}\right)}{r_{2}-m r_{1}}\right), & t \in\left[\frac{m r_{1}+r_{2}}{2}, \frac{m r_{1}+3 r_{2}}{4}\right) ; \\ \Lambda\left(\frac{4\left(t-\frac{m r_{1}+3 r_{2}}{4}\right)}{r_{2}-m r_{1}}\right), & t \in\left[\frac{m r_{1}+3 r_{2}}{4}, r_{2}\right] .\end{cases}
$$

We denote

$$
T_{f, \Lambda}\left(r_{1}, r_{2}\right):=\frac{1}{4 \Lambda(1)} \int_{m r_{1}}^{r_{2}} p_{\Lambda}(t) f^{\prime}(t) d t .
$$

Proof. Integrating by parts eq. 2.3 and changing the variables of integration, we have

$$
\begin{aligned}
& T_{f, \Lambda}\left(r_{1}, r_{2}\right)=\frac{1}{4 \Lambda(1)} \\
& \times\left[\int_{m r_{1}}^{\frac{3 m r_{1}+r_{2}}{4}} \Lambda\left(\frac{4\left(t-m r_{1}\right)}{r_{2}-m r_{1}}\right) f^{\prime}(t) d t+\int_{\frac{3 m r_{1}+r_{2}}{4}}^{\frac{m r_{1}+r_{2}}{2}} \Lambda\left(\frac{4\left(t-\frac{3 m r_{1}+r_{2}}{4}\right)}{r_{2}-m r_{1}}\right) f^{\prime}(t) d t\right. \\
& +\int_{\frac{m r_{1}+r_{2}}{2}}^{\frac{m r_{1}+3 r_{2}}{4}} \Lambda\left(\frac{4\left(t-\frac{m r_{1}+r_{2}}{2}\right)}{r_{2}-m r_{1}}\right) f^{\prime}(t) d t \\
& \left.+\int_{\frac{m r_{1}+3 r_{2}}{4}}^{r_{2}} \Lambda\left(\frac{4\left(t-\frac{m r_{1}+3 r_{2}}{4}\right)}{r_{2}-m r_{1}}\right) f^{\prime}(t) d t\right] \\
& =\frac{1}{4 \Lambda(1)}\left[\left.\Lambda\left(\frac{4\left(t-m r_{1}\right)}{r_{2}-m r_{1}}\right) f(t)\right|_{m r_{1}} ^{\frac{3 m r_{1}+r_{2}}{4}}-\int_{m r_{1}}^{\frac{3 m r_{1}+r_{2}}{4}} \frac{\varphi\left(t-m r_{1}\right)}{t-m r_{1}} f(t) d t\right. \\
& +\left.\Lambda\left(\frac{4\left(t-\frac{3 m r_{1}+r_{2}}{4}\right)}{r_{2}-m r_{1}}\right) f(t)\right|_{\frac{3 m r_{1}+r_{2}}{4}} ^{\frac{m r_{1}+r_{2}}{3}}-\int_{\frac{3 m r_{1}+r_{2}}{4}}^{\frac{m r_{1}+r_{2}}{2}} \frac{\varphi\left(t-\frac{3 m r_{1}+r_{2}}{4}\right)}{t-\frac{3 m r_{1}+r_{2}}{4}} f(t) d t \\
& +\left.\Lambda\left(\frac{4\left(t-\frac{m r_{1}+r_{2}}{2}\right)}{r_{2}-m r_{1}}\right) f(t)\right|_{\frac{m r_{1}+r_{2}}{2}} ^{\frac{m r_{1}+3 r_{2}}{2}}-\int_{\frac{m r_{1}+r_{2}}{2}}^{\frac{m r_{1}+3 r_{2}}{4}} \frac{\varphi\left(t-\frac{m r_{1}+r_{2}}{2}\right)}{t-\frac{m r_{1}+r_{2}}{2}} f(t) d t \\
& \left.+\left.\Lambda\left(\frac{4\left(t-\frac{m r_{1}+3 r_{2}}{4}\right)}{r_{2}-m r_{1}}\right) f(t)\right|_{\frac{m r_{1}+3 r_{2}}{4}} ^{r_{2}}-\int_{\frac{m r_{1}+3 r_{2}}{4}}^{r_{2}} \frac{\varphi\left(t-\frac{m r_{1}+3 r_{2}}{4}\right)}{t-\frac{m r_{1}+3 r_{2}}{4}} f(t) d t\right] \\
& =\frac{1}{4 \Lambda(1)}\left[\Lambda(1) f\left(\frac{3 m r_{1}+r_{2}}{4}\right)-{ }_{\left(\frac{3 m r_{1}+r_{2}}{4}\right)^{-}} I_{\varphi} f\left(m r_{1}\right)\right.
\end{aligned}
$$




$$
\begin{gathered}
+\Lambda(1) f\left(\frac{m r_{1}+r_{2}}{2}\right)-\left(_{\left(\frac{m r_{1}+r_{2}}{2}\right)^{-}} I_{\varphi} f\left(\frac{3 m r_{1}+r_{2}}{4}\right)\right. \\
+\Lambda(1) f\left(\frac{m r_{1}+3 r_{2}}{4}\right)-\left(\frac{m r_{1}+3 r_{2}}{4}\right)^{-} I_{\varphi} f\left(\frac{m r_{1}+r_{2}}{2}\right) \\
\left.+\Lambda(1) f(b)-b^{-} I_{\varphi} f\left(\frac{m r_{1}+3 r_{2}}{4}\right)\right] \\
=\frac{1}{4}\left[f\left(\frac{3 m r_{1}+r_{2}}{4}\right)+f\left(\frac{m r_{1}+r_{2}}{2}\right)+f\left(\frac{m r_{1}+3 r_{2}}{4}\right)+f\left(r_{2}\right)\right] \\
-\frac{1}{4 \Lambda(1)}\left[\left(\frac{3 m r_{1}+r_{2}}{4}\right)^{-} I_{\varphi} f\left(m r_{1}\right)+\left(\frac{m r_{1}+r_{2}}{2}\right)^{-} I_{\varphi} f\left(\frac{3 m r_{1}+r_{2}}{4}\right)\right. \\
+{ }_{\left.\left(\frac{m r_{1}+3 r_{2}}{4}\right)^{-} I_{\varphi} f\left(\frac{m r_{1}+r_{2}}{2}\right)+{ }_{r_{2}}^{-} I_{\varphi} f\left(\frac{m r_{1}+3 r_{2}}{4}\right)\right] .}
\end{gathered}
$$

The proof of Lemma 2.1 is completed.

Remark 2.2. Taking $\varphi(t)=t$ in Lemma 2.1, we get the following new trapezium type integral identity:

$$
\begin{gathered}
T_{f}\left(r_{1}, r_{2}\right):=\frac{1}{r_{2}-m r_{1}} \int_{m r_{1}}^{r_{2}} q(t) f^{\prime}(t) d t \\
=\frac{1}{4}\left[f\left(\frac{3 m r_{1}+r_{2}}{4}\right)+f\left(\frac{m r_{1}+r_{2}}{2}\right)+f\left(\frac{m r_{1}+3 r_{2}}{4}\right)+f\left(r_{2}\right)\right] \\
-\frac{1}{r_{2}-m r_{1}} \int_{m r_{1}}^{r_{2}} f(t) d t
\end{gathered}
$$

where

$$
q(t):= \begin{cases}t-m r_{1}, & t \in\left[m r_{1}, \frac{3 m r_{1}+r_{2}}{4}\right) \\ t-\frac{3 m r_{1}+r_{2}}{4}, & t \in\left[\frac{3 m r_{1}+r_{2}}{4}, \frac{m r_{1}+r_{2}}{2}\right) ; \\ t-\frac{m r_{1}+r_{2}}{2}, & t \in\left[\frac{m r_{1}+r_{2}}{2}, \frac{m r_{1}+3 r_{2}}{4}\right) ; \\ t-\frac{m r_{1}+3 r_{2}}{4}, & t \in\left[\frac{m r_{1}+3 r_{2}}{4}, r_{2}\right] .\end{cases}
$$

Theorem 2.3. Let $f: P \longrightarrow \mathbb{R}$ be a differentiable mapping on $\left(m r_{1}, r_{2}\right)$. If $\left|f^{\prime}\right|^{q}$ is $m$-convex on $P$ for $q>1$ and $p^{-1}+q^{-1}=1$, then the following inequality for generalized fractional integrals hold:

$$
\begin{gathered}
\left|T_{f, \Lambda}\left(r_{1}, r_{2}\right)\right| \leq \frac{\left(r_{2}-m r_{1}\right)}{16 \sqrt[q]{2} \Lambda(1)} \sqrt[p]{B_{\Lambda}(p)} \\
\times\left\{\sqrt[q]{\left|f^{\prime}\left(\frac{3 m r_{1}+r_{2}}{4}\right)\right|^{q}+m\left|f^{\prime}\left(r_{1}\right)\right|^{q}}\right. \\
+\sqrt[q]{\left|f^{\prime}\left(\frac{m r_{1}+r_{2}}{2}\right)\right|^{q}+m\left|f^{\prime}\left(\frac{3 m r_{1}+r_{2}}{4 m}\right)\right|^{q}}
\end{gathered}
$$




$$
\begin{gathered}
+\sqrt[q]{\left|f^{\prime}\left(\frac{m r_{1}+3 r_{2}}{4}\right)\right|^{q}+m\left|f^{\prime}\left(\frac{m r_{1}+r_{2}}{2 m}\right)\right|^{q}} \\
+\sqrt[q]{\left.\left|f^{\prime}\left(r_{2}\right)\right|^{q}+m\left|f^{\prime}\left(\frac{m r_{1}+3 r_{2}}{4 m}\right)\right|^{q}\right\}},
\end{gathered}
$$

where

$$
B_{\Lambda}(p):=\int_{0}^{1}[\Lambda(t)]^{p} d t
$$

Proof. From Lemma 2.1, $m$-convexity of $\left|f^{\prime}\right|^{q}$, Hölder inequality and properties of the modulus, we have

$$
\begin{aligned}
& \left|T_{f, \Lambda}\left(r_{1}, r_{2}\right)\right| \leq \frac{1}{4 \Lambda(1)} \int_{m r_{1}}^{r_{2}} p_{\Lambda}(t)\left|f^{\prime}(t)\right| d t \\
& \times\left[\int_{m r_{1}}^{\frac{3 m r_{1}+r_{2}}{4}} \Lambda\left(\frac{4\left(t-m r_{1}\right)}{r_{2}-m r_{1}}\right)\left|f^{\prime}(t)\right| d t\right. \\
& +\int_{\frac{3 m r_{1}+r_{2}}{4}}^{\frac{m r_{1}+r_{2}}{2}} \Lambda\left(\frac{4\left(t-\frac{3 m r_{1}+r_{2}}{4}\right)}{r_{2}-m r_{1}}\right)\left|f^{\prime}(t)\right| d t \\
& +\int_{\frac{m r_{1}+r_{2}}{2}}^{\frac{m r_{1}+3 r_{2}}{4}} \Lambda\left(\frac{4\left(t-\frac{m r_{1}+r_{2}}{2}\right)}{r_{2}-m r_{1}}\right)\left|f^{\prime}(t)\right| d t \\
& \left.+\int_{\frac{m r_{1}+3 r_{2}}{4}}^{r_{2}} \Lambda\left(\frac{4\left(t-\frac{m r_{1}+3 r_{2}}{4}\right)}{r_{2}-m r_{1}}\right)\left|f^{\prime}(t)\right| d t\right] \\
& =\frac{\left(r_{2}-m r_{1}\right)}{16 \Lambda(1)} \\
& \times \int_{0}^{1} \Lambda(t)\left[\left|f^{\prime}\left(\left(\frac{3 m r_{1}+r_{2}}{4}\right) t+m(1-t) r_{1}\right)\right|\right. \\
& +\left|f^{\prime}\left(\left(\frac{m r_{1}+r_{2}}{2}\right) t+\left(\frac{3 m r_{1}+r_{2}}{4}\right)(1-t)\right)\right| \\
& +\left|f^{\prime}\left(\left(\frac{m r_{1}+3 r_{2}}{4}\right) t+\left(\frac{m r_{1}+r_{2}}{2}\right)(1-t)\right)\right| \\
& \left.+\left|f^{\prime}\left(r_{2} t+\left(\frac{m r_{1}+3 r_{2}}{4}\right)(1-t)\right)\right|\right] d t \\
& \leq \frac{\left(r_{2}-m r_{1}\right)}{16 \Lambda(1)}\left(\int_{0}^{1}[\Lambda(t)]^{p} d t\right)^{\frac{1}{p}} \\
& \times\left\{\left(\int_{0}^{1}\left|f^{\prime}\left(\left(\frac{3 m r_{1}+r_{2}}{4}\right) t+m(1-t) r_{1}\right)\right|^{q} d t\right)^{\frac{1}{q}}\right.
\end{aligned}
$$




$$
\begin{aligned}
& +\left(\int_{0}^{1}\left|f^{\prime}\left(\left(\frac{m r_{1}+r_{2}}{2}\right) t+\left(\frac{3 m r_{1}+r_{2}}{4}\right)(1-t)\right)\right|^{q} d t\right)^{\frac{1}{q}} \\
& +\left(\int_{0}^{1}\left|f^{\prime}\left(\left(\frac{m r_{1}+3 r_{2}}{4}\right) t+\left(\frac{m r_{1}+r_{2}}{2}\right)(1-t)\right)\right|^{q} d t\right)^{\frac{1}{q}} \\
& \left.+\left(\int_{0}^{1}\left|f^{\prime}\left(r_{2} t+\left(\frac{m r_{1}+3 r_{2}}{4}\right)(1-t)\right)\right|^{q} d t\right)^{\frac{1}{q}}\right\} \\
& \leq \frac{\left(r_{2}-m r_{1}\right)}{16 \Lambda(1)} \sqrt[p]{B_{\Lambda}(p)} \\
& \times\left\{\left[\int_{0}^{1}\left(t\left|f^{\prime}\left(\frac{3 m r_{1}+r_{2}}{4}\right)\right|^{q}+m(1-t)\left|f^{\prime}\left(r_{1}\right)\right|^{q}\right) d t\right]^{\frac{1}{q}}\right. \\
& +\left[\int_{0}^{1}\left(t\left|f^{\prime}\left(\frac{m r_{1}+r_{2}}{2}\right)\right|^{q}+m(1-t)\left|f^{\prime}\left(\frac{3 m r_{1}+r_{2}}{4 m}\right)\right|^{q}\right) d t\right]^{\frac{1}{q}} \\
& +\left[\int_{0}^{1}\left(t\left|f^{\prime}\left(\frac{m r_{1}+3 r_{2}}{4}\right)\right|^{q}+m(1-t)\left|f^{\prime}\left(\frac{m r_{1}+r_{2}}{2 m}\right)\right|^{q}\right) d t\right]^{\frac{1}{q}} \\
& \left.+\left[\int_{0}^{1}\left(t\left|f^{\prime}\left(r_{2}\right)\right|^{q}+m(1-t)\left|f^{\prime}\left(\frac{m r_{1}+3 r_{2}}{4 m}\right)\right|^{q}\right) d t\right]^{\frac{1}{q}}\right\} \\
& =\frac{\left(r_{2}-m r_{1}\right)}{16 \sqrt[q]{2} \Lambda(1)} \sqrt[p]{B_{\Lambda}(p)} \\
& \times\left\{\sqrt[q]{\left|f^{\prime}\left(\frac{3 m r_{1}+r_{2}}{4}\right)\right|^{q}+m\left|f^{\prime}\left(r_{1}\right)\right|^{q}}\right. \\
& +\sqrt[q]{\left|f^{\prime}\left(\frac{m r_{1}+r_{2}}{2}\right)\right|^{q}+m\left|f^{\prime}\left(\frac{3 m r_{1}+r_{2}}{4 m}\right)\right|^{q}} \\
& +\sqrt[q]{\left|f^{\prime}\left(\frac{m r_{1}+3 r_{2}}{4}\right)\right|^{q}+m\left|f^{\prime}\left(\frac{m r_{1}+r_{2}}{2 m}\right)\right|^{q}} \\
& \left.+\sqrt[q]{\left|f^{\prime}\left(r_{2}\right)\right|^{q}+m\left|f^{\prime}\left(\frac{m r_{1}+3 r_{2}}{4 m}\right)\right|^{q}}\right\} .
\end{aligned}
$$

The proof of Theorem 2.3 is completed.

We point out some special cases of Theorem 2.3 . 
Corollary 2.4. Taking $m=1$ in Theorem 2.3, we get the following inequality for convex functions:

$$
\begin{gathered}
\left|T_{f, \Lambda}\left(r_{1}, r_{2}\right)\right| \leq \frac{\left(r_{2}-r_{1}\right)}{16 \sqrt[q]{2} \Lambda(1)} \sqrt[p]{B_{\Lambda}(p)} \\
\times\left\{\sqrt[q]{\left|f^{\prime}\left(\frac{3 r_{1}+r_{2}}{4}\right)\right|^{q}+\left|f^{\prime}\left(r_{1}\right)\right|^{q}}+\sqrt[q]{\left|f^{\prime}\left(\frac{r_{1}+r_{2}}{2}\right)\right|^{q}+\left|f^{\prime}\left(\frac{3 r_{1}+r_{2}}{4}\right)\right|^{q}}\right. \\
\left.+\sqrt[q]{\left|f^{\prime}\left(\frac{r_{1}+3 r_{2}}{4}\right)\right|^{q}+\left|f^{\prime}\left(\frac{r_{1}+r_{2}}{2}\right)\right|^{q}}+\sqrt[q]{\left|f^{\prime}\left(r_{2}\right)\right|^{q}+\left|f^{\prime}\left(\frac{r_{1}+3 r_{2}}{4}\right)\right|^{q}}\right\} .
\end{gathered}
$$

Corollary 2.5. Taking $p=q=2$ in Theorem 2.3, we get

$$
\begin{gathered}
\left|T_{f, \Lambda}\left(r_{1}, r_{2}\right)\right| \leq \frac{\left(r_{2}-m r_{1}\right)}{16 \sqrt{2} \Lambda(1)} \sqrt{B_{\Lambda}(2)} \\
\times\left\{\sqrt{\left|f^{\prime}\left(\frac{3 m r_{1}+r_{2}}{4}\right)\right|^{2}+m\left|f^{\prime}\left(r_{1}\right)\right|^{2}}\right. \\
+\sqrt{\left|f^{\prime}\left(\frac{m r_{1}+r_{2}}{2}\right)\right|^{2}+m\left|f^{\prime}\left(\frac{3 m r_{1}+r_{2}}{4 m}\right)\right|^{2}} \\
+\sqrt{\left|f^{\prime}\left(\frac{m r_{1}+3 r_{2}}{4}\right)\right|^{2}+m\left|f^{\prime}\left(\frac{m r_{1}+r_{2}}{2 m}\right)\right|^{2}} \\
\left.+\sqrt{\left|f^{\prime}\left(r_{2}\right)\right|^{2}+m\left|f^{\prime}\left(\frac{m r_{1}+3 r_{2}}{4 m}\right)\right|^{2}}\right\} .
\end{gathered}
$$

Corollary 2.6. Taking $K=\left\|f^{\prime}\right\|_{\infty}$ in Theorem 2.3, we get

$$
\left|T_{f, \Lambda}\left(r_{1}, r_{2}\right)\right| \leq \frac{K\left(r_{2}-m r_{1}\right)}{4 \Lambda(1)} \sqrt[q]{\frac{m+1}{2}} \sqrt[p]{B_{\Lambda}(p)} .
$$

Corollary 2.7. Taking $\varphi(t)=t$ in Theorem 2.3, we get

$$
\begin{gathered}
\left|T_{f}\left(r_{1}, r_{2}\right)\right| \leq \frac{\left(r_{2}-m r_{1}\right)}{16 \sqrt[q]{2} \sqrt[p]{p+1}} \\
\times\left\{\sqrt[q]{\left|f^{\prime}\left(\frac{3 m r_{1}+r_{2}}{4}\right)\right|^{q}+m\left|f^{\prime}\left(r_{1}\right)\right|^{q}}\right. \\
+\sqrt[q]{\left|f^{\prime}\left(\frac{m r_{1}+r_{2}}{2}\right)\right|^{q}+m\left|f^{\prime}\left(\frac{3 m r_{1}+r_{2}}{4 m}\right)\right|^{q}}
\end{gathered}
$$




$$
\begin{gathered}
+\sqrt[q]{\left|f^{\prime}\left(\frac{m r_{1}+3 r_{2}}{4}\right)\right|^{q}+m\left|f^{\prime}\left(\frac{m r_{1}+r_{2}}{2 m}\right)\right|^{q}} \\
+\sqrt[q]{\left.\left|f^{\prime}\left(r_{2}\right)\right|^{q}+m\left|f^{\prime}\left(\frac{m r_{1}+3 r_{2}}{4 m}\right)\right|^{q}\right\}} .
\end{gathered}
$$

Corollary 2.8. Taking $\varphi(t)=\frac{t^{\alpha}}{\Gamma(\alpha)}$ in Theorem 2.3, we get

$$
\begin{gathered}
\left|T_{f, \Lambda}\left(r_{1}, r_{2}\right)\right| \leq \frac{\left(r_{2}-m r_{1}\right)}{16 \sqrt[q]{2} \sqrt[p]{p \alpha+1}} \\
\times\left\{\sqrt[q]{\left|f^{\prime}\left(\frac{3 m r_{1}+r_{2}}{4}\right)\right|^{q}+m\left|f^{\prime}\left(r_{1}\right)\right|^{q}}\right. \\
+\sqrt[q]{\left|f^{\prime}\left(\frac{m r_{1}+r_{2}}{2}\right)\right|^{q}+m\left|f^{\prime}\left(\frac{3 m r_{1}+r_{2}}{4 m}\right)\right|^{q}} \\
+\sqrt[q]{\left|f^{\prime}\left(\frac{m r_{1}+3 r_{2}}{4}\right)\right|^{q}+m\left|f^{\prime}\left(\frac{m r_{1}+r_{2}}{2 m}\right)\right|^{q}} \\
\left.+\sqrt[q]{\left|f^{\prime}\left(r_{2}\right)\right|^{q}+m\left|f^{\prime}\left(\frac{m r_{1}+3 r_{2}}{4 m}\right)\right|^{q}}\right\} .
\end{gathered}
$$

Corollary 2.9. Taking $\varphi(t)=\frac{t^{\frac{\alpha}{k}}}{k \Gamma_{k}(\alpha)}$ in Theorem 2.3, we get

$$
\begin{gathered}
\left|T_{f, \Lambda}\left(r_{1}, r_{2}\right)\right| \leq \frac{\left(r_{2}-m r_{1}\right)}{16 \sqrt[q]{2} \sqrt[p]{\frac{p \alpha}{k}+1}} \\
\times\left\{\sqrt[q]{\left|f^{\prime}\left(\frac{3 m r_{1}+r_{2}}{4}\right)\right|^{q}+m\left|f^{\prime}\left(r_{1}\right)\right|^{q}}\right. \\
+\sqrt[q]{\left|f^{\prime}\left(\frac{m r_{1}+r_{2}}{2}\right)\right|^{q}+m\left|f^{\prime}\left(\frac{3 m r_{1}+r_{2}}{4 m}\right)\right|^{q}} \\
+\sqrt[q]{\left|f^{\prime}\left(\frac{m r_{1}+3 r_{2}}{4}\right)\right|^{q}+m\left|f^{\prime}\left(\frac{m r_{1}+r_{2}}{2 m}\right)\right|^{q}} \\
\left.+\sqrt[q]{\left|f^{\prime}\left(r_{2}\right)\right|^{q}+m\left|f^{\prime}\left(\frac{m r_{1}+3 r_{2}}{4 m}\right)\right|^{q}}\right\} .
\end{gathered}
$$


Corollary 2.10. Taking $\varphi(t)=t\left(r_{2}-t\right)^{\alpha-1}$ for $\alpha \in(0,1)$ in Theorem 2.3. we get

$$
\begin{gathered}
\left|T_{f, \Lambda}\left(r_{1}, r_{2}\right)\right| \leq \frac{\alpha\left(r_{2}-m r_{1}\right)}{16 \sqrt[q]{2}\left[r_{2}^{\alpha}-\left(\frac{m r_{1}+3 r_{2}}{4}\right)^{\alpha}\right]} \sqrt[p]{B_{\Lambda}^{\star}(p)} \\
\times\left\{\sqrt[q]{\left|f^{\prime}\left(\frac{3 m r_{1}+r_{2}}{4}\right)\right|^{q}+m\left|f^{\prime}\left(r_{1}\right)\right|^{q}}\right. \\
+\sqrt[q]{\left|f^{\prime}\left(\frac{m r_{1}+r_{2}}{2}\right)\right|^{q}+m\left|f^{\prime}\left(\frac{3 m r_{1}+r_{2}}{4 m}\right)\right|^{q}} \\
+\sqrt[q]{\left|f^{\prime}\left(\frac{m r_{1}+3 r_{2}}{4}\right)\right|^{q}+m\left|f^{\prime}\left(\frac{m r_{1}+r_{2}}{2 m}\right)\right|^{q}} \\
\left.+\sqrt[q]{\left|f^{\prime}\left(r_{2}\right)\right|^{q}+m\left|f^{\prime}\left(\frac{m r_{1}+3 r_{2}}{4 m}\right)\right|^{q}}\right\}
\end{gathered}
$$

where

$$
B_{\Lambda}^{\star}(p):=\frac{4}{\alpha^{p}\left(r_{2}-m r_{1}\right)} \int_{\frac{m r_{1}+3 r_{2}}{4}}^{r_{2}}\left(r_{2}^{\alpha}-t^{\alpha}\right)^{p} d t .
$$

Corollary 2.11. Taking $\varphi(t)=\frac{t}{\alpha} \exp \left[\left(-\frac{1-\alpha}{\alpha}\right) t\right]$ for $\alpha \in(0,1)$ in Theorem 2.3, we get

$$
\begin{aligned}
\left|T_{f, \Lambda}\left(r_{1}, r_{2}\right)\right| \leq & \frac{(\alpha-1)\left(r_{2}-m r_{1}\right)}{16 \sqrt[q]{2}\left\{\exp \left[\left(-\frac{1-\alpha}{\alpha}\right) \frac{\left(r_{2}-m r_{1}\right)}{4}\right]-1\right\}} \sqrt[p]{B_{\Lambda}^{\diamond}(p)} \\
& \times\left\{\sqrt[q]{\left|f^{\prime}\left(\frac{3 m r_{1}+r_{2}}{4}\right)\right|^{q}+m\left|f^{\prime}\left(r_{1}\right)\right|^{q}}\right. \\
+ & \sqrt[q]{\left|f^{\prime}\left(\frac{m r_{1}+r_{2}}{2}\right)\right|^{q}+m\left|f^{\prime}\left(\frac{3 m r_{1}+r_{2}}{4 m}\right)\right|^{q}} \\
+ & \sqrt[q]{\left|f^{\prime}\left(\frac{m r_{1}+3 r_{2}}{4}\right)\right|^{q}+m\left|f^{\prime}\left(\frac{m r_{1}+r_{2}}{2 m}\right)\right|^{q}} \\
& +\sqrt[q]{\left.\left|f^{\prime}\left(r_{2}\right)\right|^{q}+m\left|f^{\prime}\left(\frac{m r_{1}+3 r_{2}}{4 m}\right)\right|^{q}\right\}},
\end{aligned}
$$

where

$$
B_{\Lambda}^{\diamond}(p):=\frac{4 \alpha}{(\alpha-1)^{p+1}\left(r_{2}-m r_{1}\right)} \int_{0}^{\exp \left[\left(-\frac{1-\alpha}{\alpha}\right) \frac{\left(r_{2}-m r_{1}\right)}{4}\right]-1} \frac{t^{p}}{t+1} d t .
$$


Theorem 2.12. Let $f: P \longrightarrow \mathbb{R}$ be a differentiable mapping on $\left(m r_{1}, r_{2}\right)$. If $\left|f^{\prime}\right|^{q}$ is $m$-convex on $P$ for $q \geq 1$, then the following inequality for generalized fractional integrals hold:

$$
\begin{gathered}
\left|T_{f, \Lambda}\left(r_{1}, r_{2}\right)\right| \leq \frac{\left(r_{2}-m r_{1}\right)}{16 \Lambda(1)}\left[B_{\Lambda}(1)\right]^{1-\frac{1}{q}} \\
\times\left\{\sqrt[q]{C_{\Lambda}\left|f^{\prime}\left(\frac{3 m r_{1}+r_{2}}{4}\right)\right|^{q}+m\left(B_{\Lambda}(1)-C_{\Lambda}\right)\left|f^{\prime}\left(r_{1}\right)\right|^{q}}\right. \\
+\sqrt[q]{C_{\Lambda}\left|f^{\prime}\left(\frac{m r_{1}+r_{2}}{2}\right)\right|^{q}+m\left(B_{\Lambda}(1)-C_{\Lambda}\right)\left|f^{\prime}\left(\frac{3 m r_{1}+r_{2}}{4 m}\right)\right|^{q}} \\
+\sqrt[q]{C_{\Lambda}\left|f^{\prime}\left(\frac{m r_{1}+3 r_{2}}{4}\right)\right|^{q}+m\left(B_{\Lambda}(1)-C_{\Lambda}\right)\left|f^{\prime}\left(\frac{m r_{1}+r_{2}}{2 m}\right)\right|^{q}} \\
+\sqrt[q]{\left.C_{\Lambda}\left|f^{\prime}\left(r_{2}\right)\right|^{q}+m\left(B_{\Lambda}(1)-C_{\Lambda}\right)\left|f^{\prime}\left(\frac{m r_{1}+3 r_{2}}{4 m}\right)\right|^{q}\right\}},
\end{gathered}
$$

where

$$
C_{\Lambda}:=\int_{0}^{1} t[\Lambda(t)] d t
$$

and $B_{\Lambda}(1)$ is defined as in Theorem 2.3 .

Proof. From Lemma 2.1, $m$-convexity of $\left|f^{\prime}\right|^{q}$, power mean inequality and properties of the modulus, we have

$$
\begin{gathered}
\left|T_{f, \Lambda}\left(r_{1}, r_{2}\right)\right| \leq \frac{1}{4 \Lambda(1)} \int_{m r_{1}}^{r_{2}} p_{\Lambda}(t)\left|f^{\prime}(t)\right| d t \\
\times\left[\int_{m r_{1}}^{\frac{3 m r_{1}+r_{2}}{4}} \Lambda\left(\frac{4\left(t-m r_{1}\right)}{r_{2}-m r_{1}}\right)\left|f^{\prime}(t)\right| d t\right. \\
+\int_{\frac{3 m r_{1}+r_{2}}{4}}^{\frac{m r_{1}+r_{2}}{2}} \Lambda\left(\frac{4\left(t-\frac{3 m r_{1}+r_{2}}{4}\right)}{r_{2}-m r_{1}}\right)\left|f^{\prime}(t)\right| d t \\
+\int_{\frac{m r_{1}+r_{2}}{2}}^{\frac{m r_{1}+3 r_{2}}{4}} \Lambda\left(\frac{4\left(t-\frac{m r_{1}+r_{2}}{2}\right)}{r_{2}-m r_{1}}\right)\left|f^{\prime}(t)\right| d t \\
\left.+\int_{\frac{m r_{1}+3 r_{2}}{4}}^{r_{2}}\left(\frac{4\left(t-\frac{m r_{1}+3 r_{2}}{4}\right)}{r_{2}-m r_{1}}\right)\left|f^{\prime}(t)\right| d t\right] \\
\times \int_{0}^{1} \Lambda(t)\left[\left|f^{\prime}\left(\left(\frac{3 m r_{1}+r_{2}}{4}\right) t+m(1-t) r_{1}\right)\right|\right.
\end{gathered}
$$




$$
\begin{aligned}
& +\left|f^{\prime}\left(\left(\frac{m r_{1}+r_{2}}{2}\right) t+\left(\frac{3 m r_{1}+r_{2}}{4}\right)(1-t)\right)\right| \\
& +\left|f^{\prime}\left(\left(\frac{m r_{1}+3 r_{2}}{4}\right) t+\left(\frac{m r_{1}+r_{2}}{2}\right)(1-t)\right)\right| \\
& \left.+\left|f^{\prime}\left(r_{2} t+\left(\frac{m r_{1}+3 r_{2}}{4}\right)(1-t)\right)\right|\right] d t \\
& \leq \frac{\left(r_{2}-m r_{1}\right)}{16 \Lambda(1)}\left(\int_{0}^{1} \Lambda(t) d t\right)^{1-\frac{1}{q}} \\
& \times\left\{\left(\int_{0}^{1} \Lambda(t)\left|f^{\prime}\left(\left(\frac{3 m r_{1}+r_{2}}{4}\right) t+m(1-t) r_{1}\right)\right|^{q} d t\right)^{\frac{1}{q}}\right. \\
& +\left(\int_{0}^{1} \Lambda(t)\left|f^{\prime}\left(\left(\frac{m r_{1}+r_{2}}{2}\right) t+\left(\frac{3 m r_{1}+r_{2}}{4}\right)(1-t)\right)\right|^{q} d t\right)^{\frac{1}{q}} \\
& +\left(\int_{0}^{1} \Lambda(t)\left|f^{\prime}\left(\left(\frac{m r_{1}+3 r_{2}}{4}\right) t+\left(\frac{m r_{1}+r_{2}}{2}\right)(1-t)\right)\right|^{q} d t\right)^{\frac{1}{q}} \\
& \left.+\left(\int_{0}^{1} \Lambda(t)\left|f^{\prime}\left(r_{2} t+\left(\frac{m r_{1}+3 r_{2}}{4}\right)(1-t)\right)\right|^{q} d t\right)^{\frac{1}{q}}\right\} \\
& \leq \frac{\left(r_{2}-m r_{1}\right)}{16 \Lambda(1)}\left[B_{\Lambda}(1)\right]^{1-\frac{1}{q}} \\
& \times\left\{\left[\int_{0}^{1} \Lambda(t)\left(t\left|f^{\prime}\left(\frac{3 m r_{1}+r_{2}}{4}\right)\right|^{q}+m(1-t)\left|f^{\prime}\left(r_{1}\right)\right|^{q}\right) d t\right]^{\frac{1}{q}}\right. \\
& +\left[\int_{0}^{1} \Lambda(t)\left(t\left|f^{\prime}\left(\frac{m r_{1}+r_{2}}{2}\right)\right|^{q}+m(1-t)\left|f^{\prime}\left(\frac{3 m r_{1}+r_{2}}{4 m}\right)\right|^{q}\right) d t\right]^{\frac{1}{q}} \\
& +\left[\int_{0}^{1} \Lambda(t)\left(t\left|f^{\prime}\left(\frac{m r_{1}+3 r_{2}}{4}\right)\right|^{q}+m(1-t)\left|f^{\prime}\left(\frac{m r_{1}+r_{2}}{2 m}\right)\right|^{q}\right) d t\right]^{\frac{1}{q}} \\
& \left.+\left[\int_{0}^{1} \Lambda(t)\left(t\left|f^{\prime}\left(r_{2}\right)\right|^{q}+m(1-t)\left|f^{\prime}\left(\frac{m r_{1}+3 r_{2}}{4 m}\right)\right|^{q}\right) d t\right]^{\frac{1}{q}}\right\} \\
& =\frac{\left(r_{2}-m r_{1}\right)}{16 \Lambda(1)}\left[B_{\Lambda}(1)\right]^{1-\frac{1}{q}} \\
& \times\left\{\sqrt[q]{C_{\Lambda}\left|f^{\prime}\left(\frac{3 m r_{1}+r_{2}}{4}\right)\right|^{q}+m\left(B_{\Lambda}(1)-C_{\Lambda}\right)\left|f^{\prime}\left(r_{1}\right)\right|^{q}}\right. \\
& +\sqrt[q]{C_{\Lambda}\left|f^{\prime}\left(\frac{m r_{1}+r_{2}}{2}\right)\right|^{q}+m\left(B_{\Lambda}(1)-C_{\Lambda}\right)\left|f^{\prime}\left(\frac{3 m r_{1}+r_{2}}{4 m}\right)\right|^{q}}
\end{aligned}
$$




$$
\begin{gathered}
+\sqrt[q]{C_{\Lambda}\left|f^{\prime}\left(\frac{m r_{1}+3 r_{2}}{4}\right)\right|^{q}+m\left(B_{\Lambda}(1)-C_{\Lambda}\right)\left|f^{\prime}\left(\frac{m r_{1}+r_{2}}{2 m}\right)\right|^{q}} \\
+\sqrt[q]{\left.C_{\Lambda}\left|f^{\prime}\left(r_{2}\right)\right|^{q}+m\left(B_{\Lambda}(1)-C_{\Lambda}\right)\left|f^{\prime}\left(\frac{m r_{1}+3 r_{2}}{4 m}\right)\right|^{q}\right\} .}
\end{gathered}
$$

The proof of Theorem 2.12 is completed.

We point out some special cases of Theorem 2.12 .

Corollary 2.13. Taking $m=1$ in Theorem 2.12, we get the following inequality for convex functions:

$$
\begin{gathered}
\left|T_{f, \Lambda}\left(r_{1}, r_{2}\right)\right| \leq \frac{\left(r_{2}-r_{1}\right)}{16 \Lambda(1)}\left[B_{\Lambda}(1)\right]^{1-\frac{1}{q}} \\
\times\left\{\sqrt[q]{C_{\Lambda}\left|f^{\prime}\left(\frac{3 r_{1}+r_{2}}{4}\right)\right|^{q}+\left(B_{\Lambda}(1)-C_{\Lambda}\right)\left|f^{\prime}\left(r_{1}\right)\right|^{q}}\right. \\
+\sqrt[q]{C_{\Lambda}\left|f^{\prime}\left(\frac{r_{1}+r_{2}}{2}\right)\right|^{q}+\left(B_{\Lambda}(1)-C_{\Lambda}\right)\left|f^{\prime}\left(\frac{3 r_{1}+r_{2}}{4}\right)\right|^{q}} \\
+\sqrt[q]{C_{\Lambda}\left|f^{\prime}\left(\frac{r_{1}+3 r_{2}}{4}\right)\right|^{q}+\left(B_{\Lambda}(1)-C_{\Lambda}\right)\left|f^{\prime}\left(\frac{r_{1}+r_{2}}{2}\right)\right|^{q}} \\
\left.+\sqrt[q]{C_{\Lambda}\left|f^{\prime}\left(r_{2}\right)\right|^{q}+\left(B_{\Lambda}(1)-C_{\Lambda}\right)\left|f^{\prime}\left(\frac{r_{1}+3 r_{2}}{4}\right)\right|^{q}}\right\} .
\end{gathered}
$$

Corollary 2.14. Taking $q=1$ in Theorem 2.12, we get

$$
\begin{gathered}
\left|T_{f, \Lambda}\left(r_{1}, r_{2}\right)\right| \leq \frac{\left(r_{2}-m r_{1}\right)}{16 \Lambda(1)} \\
\times\left\{C_{\Lambda}\left|f^{\prime}\left(\frac{3 m r_{1}+r_{2}}{4}\right)\right|+m\left(B_{\Lambda}(1)-C_{\Lambda}\right)\left|f^{\prime}\left(r_{1}\right)\right|\right. \\
+C_{\Lambda}\left|f^{\prime}\left(\frac{m r_{1}+r_{2}}{2}\right)\right|+m\left(B_{\Lambda}(1)-C_{\Lambda}\right)\left|f^{\prime}\left(\frac{3 m r_{1}+r_{2}}{4 m}\right)\right| \\
+C_{\Lambda}\left|f^{\prime}\left(\frac{m r_{1}+3 r_{2}}{4}\right)\right|+m\left(B_{\Lambda}(1)-C_{\Lambda}\right)\left|f^{\prime}\left(\frac{m r_{1}+r_{2}}{2 m}\right)\right| \\
\left.+C_{\Lambda}\left|f^{\prime}\left(r_{2}\right)\right|+m\left(B_{\Lambda}(1)-C_{\Lambda}\right)\left|f^{\prime}\left(\frac{m r_{1}+3 r_{2}}{4 m}\right)\right|\right\} .
\end{gathered}
$$

Corollary 2.15. Taking $K=\left\|f^{\prime}\right\|_{\infty}$ in Theorem 2.12, we get

$$
\left|T_{f, \Lambda}\left(r_{1}, r_{2}\right)\right| \leq \frac{K\left(r_{2}-m r_{1}\right)}{4 \Lambda(1)}\left[B_{\Lambda}(1)\right]^{1-\frac{1}{q}} \times \sqrt[q]{C_{\Lambda}+m\left(B_{\Lambda}(1)-C_{\Lambda}\right)} .
$$


Corollary 2.16. Taking $\varphi(t)=t$ in Theorem 2.12, we get

$$
\begin{gathered}
\left|T_{f}\left(r_{1}, r_{2}\right)\right| \leq \frac{\left(r_{2}-m r_{1}\right)}{32 \sqrt[q]{3}} \\
\times\left\{\sqrt[q]{2\left|f^{\prime}\left(\frac{3 m r_{1}+r_{2}}{4}\right)\right|^{q}+m\left|f^{\prime}\left(r_{1}\right)\right|^{q}}\right. \\
+\sqrt[q]{2\left|f^{\prime}\left(\frac{m r_{1}+r_{2}}{2}\right)\right|^{q}+m\left|f^{\prime}\left(\frac{3 m r_{1}+r_{2}}{4 m}\right)\right|^{q}} \\
+\sqrt[q]{2\left|f^{\prime}\left(\frac{m r_{1}+3 r_{2}}{4}\right)\right|^{q}+m\left|f^{\prime}\left(\frac{m r_{1}+r_{2}}{2 m}\right)\right|^{q}} \\
\left.+\sqrt[q]{2\left|f^{\prime}\left(r_{2}\right)\right|^{q}+m\left|f^{\prime}\left(\frac{m r_{1}+3 r_{2}}{4 m}\right)\right|^{q}}\right\} .
\end{gathered}
$$

Corollary 2.17. Taking $\varphi(t)=\frac{t^{\alpha}}{\Gamma(\alpha)}$ in Theorem 2.12, we get

$$
\begin{gathered}
\left|T_{f, \Lambda}\left(r_{1}, r_{2}\right)\right| \leq \frac{\left(r_{2}-m r_{1}\right)}{16 \sqrt[q]{\alpha+2}} \frac{\Gamma(\alpha+1)}{\Gamma(\alpha+2)} \\
\times\left\{\sqrt[q]{(\alpha+1)\left|f^{\prime}\left(\frac{3 m r_{1}+r_{2}}{4}\right)\right|^{q}+m\left|f^{\prime}\left(r_{1}\right)\right|^{q}}\right. \\
+\sqrt[q]{(\alpha+1)\left|f^{\prime}\left(\frac{m r_{1}+r_{2}}{2}\right)\right|^{q}+m\left|f^{\prime}\left(\frac{3 m r_{1}+r_{2}}{4 m}\right)\right|^{q}} \\
+\sqrt[q]{(\alpha+1)\left|f^{\prime}\left(\frac{m r_{1}+3 r_{2}}{4}\right)\right|^{q}+m\left|f^{\prime}\left(\frac{m r_{1}+r_{2}}{2 m}\right)\right|^{q}} \\
\left.+\sqrt[q]{(\alpha+1)\left|f^{\prime}\left(r_{2}\right)\right|^{q}+m\left|f^{\prime}\left(\frac{m r_{1}+3 r_{2}}{4 m}\right)\right|^{q}}\right\} .
\end{gathered}
$$

Corollary 2.18. Taking $\varphi(t)=\frac{t^{\frac{\alpha}{k}}}{k \Gamma_{k}(\alpha)}$ in Theorem 2.12, we get

$$
\begin{gathered}
\left|T_{f, \Lambda}\left(r_{1}, r_{2}\right)\right| \leq \frac{\left(r_{2}-m r_{1}\right)}{16 \sqrt[q]{\frac{\alpha}{k}+2}} \frac{\Gamma_{k}(\alpha+k)}{\Gamma_{k}(\alpha+k+1)} \\
\times\left\{\sqrt[q]{\left(\frac{\alpha}{k}+1\right)\left|f^{\prime}\left(\frac{3 m r_{1}+r_{2}}{4}\right)\right|^{q}+m\left|f^{\prime}\left(r_{1}\right)\right|^{q}}\right. \\
+\sqrt[q]{\left(\frac{\alpha}{k}+1\right)\left|f^{\prime}\left(\frac{m r_{1}+r_{2}}{2}\right)\right|^{q}+m\left|f^{\prime}\left(\frac{3 m r_{1}+r_{2}}{4 m}\right)\right|^{q}}
\end{gathered}
$$




$$
\begin{gathered}
+\sqrt[q]{\left(\frac{\alpha}{k}+1\right)\left|f^{\prime}\left(\frac{m r_{1}+3 r_{2}}{4}\right)\right|^{q}+m\left|f^{\prime}\left(\frac{m r_{1}+r_{2}}{2 m}\right)\right|^{q}} \\
+\sqrt[q]{\left.\left(\frac{\alpha}{k}+1\right)\left|f^{\prime}\left(r_{2}\right)\right|^{q}+m\left|f^{\prime}\left(\frac{m r_{1}+3 r_{2}}{4 m}\right)\right|^{q}\right\} .}
\end{gathered}
$$

Corollary 2.19. Taking $\varphi(t)=t\left(r_{2}-t\right)^{\alpha-1}$ for $\alpha \in(0,1)$ in Theorem 2.12. we get

$$
\begin{gathered}
\left|T_{f, \Lambda}\left(r_{1}, r_{2}\right)\right| \leq \frac{\left(r_{2}-m r_{1}\right)}{16 \Lambda^{*}(1)}\left[B_{\Lambda}(1)\right]^{1-\frac{1}{q}} \\
\times\left\{\sqrt[q]{C_{\Lambda^{*}}\left|f^{\prime}\left(\frac{3 m r_{1}+r_{2}}{4}\right)\right|^{q}+m\left(B_{\Lambda}(1)-C_{\Lambda^{*}}\right)\left|f^{\prime}\left(r_{1}\right)\right|^{q}}\right. \\
+\sqrt[q]{C_{\Lambda^{*}}\left|f^{\prime}\left(\frac{m r_{1}+r_{2}}{2}\right)\right|^{q}+m\left(B_{\Lambda}(1)-C_{\Lambda^{*}}\right)\left|f^{\prime}\left(\frac{3 m r_{1}+r_{2}}{4 m}\right)\right|^{q}} \\
+\sqrt[q]{C_{\Lambda^{*}}\left|f^{\prime}\left(\frac{m r_{1}+3 r_{2}}{4}\right)\right|^{q}+m\left(B_{\Lambda}(1)-C_{\Lambda^{*}}\right)\left|f^{\prime}\left(\frac{m r_{1}+r_{2}}{2 m}\right)\right|^{q}} \\
+\sqrt[q]{\left.C_{\Lambda^{*}}\left|f^{\prime}\left(r_{2}\right)\right|^{q}+m\left(B_{\Lambda}(1)-C_{\Lambda^{*}}\right)\left|f^{\prime}\left(\frac{m r_{1}+3 r_{2}}{4 m}\right)\right|^{q}\right\}},
\end{gathered}
$$

where

$$
\Lambda^{*}(t):=\frac{r_{2}^{\alpha}-\left(r_{2}-\frac{\left(r_{2}-m r_{1}\right) t}{4}\right)^{\alpha}}{\alpha}, \quad C_{\Lambda^{*}}:=\int_{0}^{1} t\left[\Lambda^{*}(t)\right] d t
$$

and

$$
B_{\Lambda}(1):=\frac{4}{\alpha\left(r_{2}-m r_{1}\right)}\left\{r_{2}^{\alpha}\left(\frac{m r_{1}+3 r_{2}}{4}\right)-\frac{r_{2}^{\alpha+1}-\left(\frac{m r_{1}+3 r_{2}}{4}\right)^{\alpha+1}}{\alpha+1}\right\} .
$$

Corollary 2.20. Taking $\varphi(t)=\frac{t}{\alpha} \exp \left[\left(-\frac{1-\alpha}{\alpha}\right) t\right]$ for $\alpha \in(0,1)$ in Theorem 2.12, we get

$$
\begin{gathered}
\left|T_{f, \Lambda}\left(r_{1}, r_{2}\right)\right| \leq \frac{\left(r_{2}-m r_{1}\right)}{16 \Lambda^{\diamond}(1)}\left[B_{\Lambda}^{\diamond}(1)\right]^{1-\frac{1}{q}} \\
\times\left\{\sqrt[q]{C_{\Lambda^{\diamond}}\left|f^{\prime}\left(\frac{3 m r_{1}+r_{2}}{4}\right)\right|^{q}+m\left(B_{\Lambda}^{\diamond}(1)-C_{\Lambda^{\diamond}}\right)\left|f^{\prime}\left(r_{1}\right)\right|^{q}}\right. \\
+\sqrt[q]{C_{\Lambda^{\diamond}}\left|f^{\prime}\left(\frac{m r_{1}+r_{2}}{2}\right)\right|^{q}+m\left(B_{\Lambda}^{\diamond}(1)-C_{\Lambda^{\diamond}}\right)\left|f^{\prime}\left(\frac{3 m r_{1}+r_{2}}{4 m}\right)\right|^{q}} \\
+\sqrt[q]{C_{\Lambda^{\diamond}}\left|f^{\prime}\left(\frac{m r_{1}+3 r_{2}}{4}\right)\right|^{q}+m\left(B_{\Lambda}^{\diamond}(1)-C_{\Lambda^{\diamond}}\right)\left|f^{\prime}\left(\frac{m r_{1}+r_{2}}{2 m}\right)\right|^{q}}
\end{gathered}
$$




$$
\left.+\sqrt[q]{C_{\Lambda^{\diamond}}\left|f^{\prime}\left(r_{2}\right)\right|^{q}+m\left(B_{\Lambda}^{\diamond}(1)-C_{\Lambda^{\diamond}}\right)\left|f^{\prime}\left(\frac{m r_{1}+3 r_{2}}{4 m}\right)\right|^{q}}\right\},
$$

where

$$
\Lambda^{\diamond}(t):=\frac{\exp \left[\left(-\frac{1-\alpha}{\alpha}\right) \frac{\left(r_{2}-m r_{1}\right) t}{4}\right]-1}{\alpha-1}, \quad C_{\Lambda^{\diamond}}:=\int_{0}^{1} t\left[\Lambda^{\diamond}(t)\right] d t
$$

and $B_{\Lambda}^{\diamond}(1)$ is defined by eq. (2.16) for $p=1$.

\section{Applications}

Consider the following special means for different real numbers $r_{1}, r_{2}$ and $r_{1} r_{2} \neq 0$, as follows:

(1) The arithmetic mean:

$$
A:=A\left(r_{1}, r_{2}\right)=\frac{r_{1}+r_{2}}{2},
$$

(2) The harmonic mean:

$$
H:=H\left(r_{1}, r_{2}\right)=\frac{2}{\frac{1}{r_{1}}+\frac{1}{r_{2}}},
$$

(3) The logarithmic mean:

$$
L:=L\left(r_{1}, r_{2}\right)=\frac{r_{2}-r_{1}}{\ln \left|r_{2}\right|-\ln \left|r_{1}\right|},
$$

(4) The generalized log-mean:

$$
L_{r}:=L_{r}\left(r_{1}, r_{2}\right)=\left[\frac{r_{2}^{r+1}-r_{1}^{r+1}}{(r+1)\left(r_{2}-r_{1}\right)}\right]^{\frac{1}{r}} ; r \in \mathbb{Z} \backslash\{-1,0\} .
$$

It is well known that $L_{r}$ is monotonic nondecreasing over $r \in \mathbb{Z}$ with $L_{-1}:=$ $L$. In particular, we have the following inequality $H \leq L \leq A$. Now, using the theory results in Section 2, we give some applications to special means for different real numbers.

Proposition 3.1. Let $r_{1}, r_{2} \in \mathbb{R} \backslash\{0\}$, where $r_{1}<r_{2}$. Then for $r \in \mathbb{N}$ and $r \geq 2$, where $q>1$ and $p^{-1}+q^{-1}=1$, the following inequality hold:

$$
\begin{gathered}
\left|A\left(\frac{A\left(A^{r}\left(3 r_{1}, r_{2}\right), A^{r}\left(r_{1}, 3 r_{2}\right)\right)}{2^{r}}, A\left(A\left(r_{1}, r_{2}\right), r_{2}^{r}\right)\right)-L_{r}^{r}\left(r_{1}, r_{2}\right)\right| \\
\leq \frac{r\left(r_{2}-r_{1}\right)}{16 \sqrt[p]{p+1}} \\
\times\left\{\sqrt[q]{A\left(\left|r_{1}\right|^{q(r-1)},\left|\frac{3 r_{1}+r_{2}}{4}\right|^{q(r-1)}\right)}+\sqrt[q]{A\left(\left|\frac{r_{1}+r_{2}}{2}\right|^{q(r-1)},\left|\frac{3 r_{1}+r_{2}}{4}\right|^{q(r-1)}\right)}\right.
\end{gathered}
$$


$\left.+\sqrt[q]{A\left(\left|\frac{r_{1}+3 r_{2}}{4}\right|^{q(r-1)},\left|\frac{r_{1}+r_{2}}{2}\right|^{q(r-1)}\right)}+\sqrt[q]{A\left(\left|\frac{r_{1}+3 r_{2}}{4}\right|^{q(r-1)},\left|r_{2}\right|^{q(r-1)}\right)}\right\}$.

Proof. Taking $m=1, f(t)=t^{r}$ and $\varphi(t)=t$, in Theorem 2.3, one can obtain the result immediately.

Proposition 3.2. Let $r_{1}, r_{2} \in \mathbb{R} \backslash\{0\}$, where $r_{1}<r_{2}$. Then for $q>1$ and $p^{-1}+q^{-1}=1$, the following inequality hold:

$$
\begin{aligned}
& \left|\frac{1}{H\left(H\left(\frac{3 r_{1}+r_{2}}{4}, \frac{r_{1}+r_{2}}{2}\right), H\left(\frac{r_{1}+3 r_{2}}{4}, r_{2}\right)\right)}-\frac{1}{L\left(r_{1}, r_{2}\right)}\right| \leq \frac{\left(r_{2}-r_{1}\right)}{16 \sqrt[p]{p+1}} \\
& \times\left\{\frac{1}{\sqrt[q]{H\left(\left|r_{1}\right|^{2 q},\left|\frac{3 r_{1}+r_{2}}{4}\right|^{2 q}\right)}}+\frac{1}{\sqrt[q]{H\left(\left|\frac{r_{1}+r_{2}}{2}\right|^{2 q},\left|\frac{3 r_{1}+r_{2}}{4}\right|^{2 q}\right)}}\right. \\
& \left.+\frac{1}{\sqrt[q]{H\left(\left|\frac{r_{1}+3 r_{2}}{4}\right|^{2 q},\left|\frac{r_{1}+r_{2}}{2}\right|^{2 q}\right)}}+\frac{1}{\sqrt[q]{H\left(\left|\frac{r_{1}+3 r_{2}}{4}\right|^{2 q},\left|r_{2}\right|^{2 q}\right)}}\right\} .
\end{aligned}
$$

Proof. Taking $m=1, f(t)=\frac{1}{t}$ and $\varphi(t)=t$, in Theorem 2.3, one can obtain the result immediately.

Proposition 3.3. Let $r_{1}, r_{2} \in \mathbb{R} \backslash\{0\}$, where $r_{1}<r_{2}$. Then for $r \in \mathbb{N}$ and $r \geq 2$, where $q \geq 1$, the following inequality hold:

$$
\begin{aligned}
\mid A\left(\frac{A\left(A^{r}\left(3 r_{1}, r_{2}\right), A^{r}\left(r_{1}, 3 r_{2}\right)\right)}{\left.2^{r}, A\left(A\left(r_{1}, r_{2}\right), r_{2}^{r}\right)\right)-L_{r}^{r}\left(r_{1}, r_{2}\right) \mid}\right. & \leq \sqrt[q]{\frac{2}{3} \frac{r\left(r_{2}-r_{1}\right)}{32}} \\
& \times\left\{\sqrt[q]{A\left(\left.\left|r_{1}\right|\right|^{(r-1)}, 2\left|\frac{3 r_{1}+r_{2}}{4}\right|^{q(r-1)}\right)}\right. \\
+ & \sqrt[q]{A\left(2\left|\frac{r_{1}+r_{2}}{2}\right|^{q(r-1)},\left|\frac{3 r_{1}+r_{2}}{4}\right|^{q(r-1)}\right)} \\
+ & \sqrt[q]{A\left(2\left|\frac{r_{1}+3 r_{2}}{4}\right|^{q(r-1)},\left|\frac{r_{1}+r_{2}}{2}\right|^{q(r-1)}\right)} \\
& +\sqrt[q]{\left.A\left(\left|\frac{r_{1}+3 r_{2}}{4}\right|^{q(r-1)}, 2\left|r_{2}\right|^{q(r-1)}\right)\right\}} .
\end{aligned}
$$

Proof. Taking $m=1, f(t)=t^{r}$ and $\varphi(t)=t$, in Theorem 2.12, one can obtain the result immediately. 
Proposition 3.4. Let $r_{1}, r_{2} \in \mathbb{R} \backslash\{0\}$, where $r_{1}<r_{2}$. Then for $q \geq 1$, the following inequality hold:

$$
\begin{aligned}
& \left|\frac{1}{H\left(H\left(\frac{3 r_{1}+r_{2}}{4}, \frac{r_{1}+r_{2}}{2}\right), H\left(\frac{r_{1}+3 r_{2}}{4}, r_{2}\right)\right)}-\frac{1}{L\left(r_{1}, r_{2}\right)}\right| \leq \sqrt[q]{\frac{2}{3}} \frac{\left(r_{2}-r_{1}\right)}{32} \\
& \times\left\{\frac{1}{\sqrt[q]{H\left(2\left|r_{1}\right|^{2 q},\left|\frac{3 r_{1}+r_{2}}{4}\right|^{2 q}\right)}}+\frac{1}{\sqrt[q]{H\left(\left|\frac{r_{1}+r_{2}}{2}\right|^{2 q}, 2\left|\frac{3 r_{1}+r_{2}}{4}\right|^{2 q}\right)}}\right. \\
& \left.+\frac{1}{\sqrt[q]{H\left(\left|\frac{r_{1}+3 r_{2}}{4}\right|^{2 q}, 2\left|\frac{r_{1}+r_{2}}{2}\right|^{2 q}\right)}}+\frac{1}{\sqrt[q]{H\left(2\left|\frac{r_{1}+3 r_{2}}{4}\right|^{2 q},\left|r_{2}\right|^{2 q}\right)}}\right\} .
\end{aligned}
$$

Proof. Taking $m=1, f(t)=\frac{1}{t}$ and $\varphi(t)=t$, in Theorem 2.12 one can obtain the result immediately.

Remark 3.5. Applying our Theorems 2.3 and 2.12 for special values $m$ and various suitable choices of function $\varphi(t)=\frac{t^{\alpha}}{\Gamma(\alpha)}, \frac{t^{\frac{\alpha}{k}}}{k \Gamma_{k}(\alpha)}, \varphi(t)=t\left(r_{2}-t\right)^{\alpha-1}$ and $\varphi(t)=\frac{t}{\alpha} \exp \left[\left(-\frac{1-\alpha}{\alpha}\right) t\right]$ for $\alpha \in(0,1)$, such that $\left|f^{\prime}\right|^{q}$ to be $m$-convex, we can deduce some new general fractional integral inequalities using special means. The details are left to the interested reader.

Next, we provide some new error estimates for the trapezium formula. Let $Q$ be the partition of the points $r_{1}=x_{0}<x_{1}<\ldots<x_{k}=r_{2}$ of the interval $\left[r_{1}, r_{2}\right]$. Let consider the following quadrature formula:

$$
\int_{r_{1}}^{r_{2}} f(x) d x=T(f, Q)+E(f, Q)
$$

where

$$
\begin{aligned}
T(f, Q)=\sum_{i=0}^{k-1}\left[f\left(\frac{3 x_{i}+x_{i+1}}{4}\right)\right. & \left.+f\left(\frac{x_{i}+x_{i+1}}{2}\right)+f\left(\frac{x_{i}+3 x_{i+1}}{4}\right)+f\left(x_{i+1}\right)\right] \\
& \times \frac{\left(x_{i+1}-x_{i}\right)}{4}
\end{aligned}
$$

is the trapezium version and $E(f, Q)$ is denote their associated approximation error.

Proposition 3.6. Let $f:\left[r_{1}, r_{2}\right] \longrightarrow \mathbb{R}$ be a differentiable function on $\left(r_{1}, r_{2}\right)$, where $r_{1}<r_{2}$. If $\left|f^{\prime}\right|^{q}$ is convex on $\left[r_{1}, r_{2}\right]$ for $q>1$ and $p^{-1}+q^{-1}=$ 1 , then the following inequality holds:

$$
|E(f, Q)| \leq \frac{1}{16 \sqrt[q]{2} \sqrt[p]{p+1}} \times \sum_{i=0}^{k-1}\left(x_{i+1}-x_{i}\right)^{2}
$$




$$
\begin{gathered}
\times\left\{\sqrt[q]{\left.\left|f^{\prime}\left(\frac{3 x_{i}+x_{i+1}}{4}\right)\right|\right|^{q}+\left|f^{\prime}\left(x_{i}\right)\right|^{q}}\right. \\
+\sqrt[q]{\left|f^{\prime}\left(\frac{x_{i}+x_{i+1}}{2}\right)\right|^{q}+\left|f^{\prime}\left(\frac{3 x_{i}+x_{i+1}}{4}\right)\right|^{q}} \\
+\sqrt[q]{\left|f^{\prime}\left(\frac{x_{i}+3 x_{i+1}}{4}\right)\right|^{q}+\left|f^{\prime}\left(\frac{x_{i}+x_{i+1}}{2}\right)\right|^{q}} \\
+\sqrt[q]{\left.\left|f^{\prime}\left(\frac{x_{i}+3 x_{i+1}}{4}\right)\right|^{q}+\left|f^{\prime}\left(x_{i+1}\right)\right|^{q}\right\}} .
\end{gathered}
$$

Proof. Applying Theorem 2.3 for $m=1$ for $\varphi(t)=t$ on the subintervals $\left[x_{i}, x_{i+1}\right](i=0, \ldots, k-1)$ of the partition $Q$, we have

$$
\begin{gathered}
\mid f\left(\frac{3 x_{i}+x_{i+1}}{4}\right)+f\left(\frac{x_{i}+x_{i+1}}{2}\right)+f\left(\frac{x_{i}+3 x_{i+1}}{4}\right)+f\left(x_{i+1}\right) \\
-\frac{4}{x_{i+1}-x_{i}} \int_{x_{i}}^{x_{i+1}} f(x) d x \mid \\
\leq \frac{\left(x_{i+1}-x_{i}\right)}{4 \sqrt[q]{2} \sqrt[p]{p+1}} \\
\times\left\{\sqrt[q]{\left|f^{\prime}\left(\frac{3 x_{i}+x_{i+1}}{4}\right)\right|^{q}+\left|f^{\prime}\left(x_{i}\right)\right|^{q}}\right. \\
+\sqrt[q]{\left|f^{\prime}\left(\frac{x_{i}+x_{i+1}}{2}\right)\right|^{q}+\left|f^{\prime}\left(\frac{3 x_{i}+x_{i+1}}{4}\right)\right|^{q}} \\
+\sqrt[q]{\left|f^{\prime}\left(\frac{x_{i}+3 x_{i+1}}{4}\right)\right|^{q}+\left|f^{\prime}\left(\frac{x_{i}+x_{i+1}}{2}\right)\right|^{q}} \\
+\sqrt[q]{\left.\left|f^{\prime}\left(\frac{x_{i}+3 x_{i+1}}{4}\right)\right|^{q}+\left|f^{\prime}\left(x_{i+1}\right)\right|^{q}\right\}} .
\end{gathered}
$$

Hence from (3.6), we get

$$
\begin{gathered}
|E(f, Q)|=\left|\int_{r_{1}}^{r_{2}} f(x) d x-T(f, Q)\right| \leq \mid \sum_{i=0}^{k-1}\left\{\int_{x_{i}}^{x_{i+1}} f(x) d x\right. \\
\left.-\left[f\left(\frac{3 x_{i}+x_{i+1}}{4}\right)+f\left(\frac{x_{i}+x_{i+1}}{2}\right)+f\left(\frac{x_{i}+3 x_{i+1}}{4}\right)+f\left(x_{i+1}\right)\right] \frac{\left(x_{i+1}-x_{i}\right)}{4}\right\} \mid \\
\leq \sum_{i=0}^{k-1} \mid\left\{\int_{x_{i}}^{x_{i+1}} f(x) d x\right.
\end{gathered}
$$


50

$$
\begin{aligned}
&-\left[f\left(\frac{3 x_{i}+x_{i+1}}{4}\right)+\right.\left.\left.f\left(\frac{x_{i}+x_{i+1}}{2}\right)+f\left(\frac{x_{i}+3 x_{i+1}}{4}\right)+f\left(x_{i+1}\right)\right] \frac{\left(x_{i+1}-x_{i}\right)}{4}\right\} \mid \\
& \leq \frac{1}{16 \sqrt[q]{2} \sqrt[p]{p+1}} \times \sum_{i=0}^{k-1}\left(x_{i+1}-x_{i}\right)^{2} \\
& \times\left\{\sqrt[q]{\left|f^{\prime}\left(\frac{3 x_{i}+x_{i+1}}{4}\right)\right|^{q}+\left|f^{\prime}\left(x_{i}\right)\right|^{q}}\right. \\
&+ \sqrt[q]{\left|f^{\prime}\left(\frac{x_{i}+x_{i+1}}{2}\right)\right|^{q}+\left|f^{\prime}\left(\frac{3 x_{i}+x_{i+1}}{4}\right)\right|^{q}} \\
&+ \sqrt[q]{\left|f^{\prime}\left(\frac{x_{i}+3 x_{i+1}}{4}\right)\right|^{q}+\left|f^{\prime}\left(\frac{x_{i}+x_{i+1}}{2}\right)\right|^{q}} \\
&+\sqrt[q]{\left.\left|f^{\prime}\left(\frac{x_{i}+3 x_{i+1}}{4}\right)\right|^{q}+\left|f^{\prime}\left(x_{i+1}\right)\right|^{q}\right\}} .
\end{aligned}
$$

The proof of Proposition 3.6 is completed.

Proposition 3.7. Let $f:\left[r_{1}, r_{2}\right] \longrightarrow \mathbb{R}$ be a differentiable function on $\left(r_{1}, r_{2}\right)$, where $r_{1}<r_{2}$. If $\left|f^{\prime}\right|^{q}$ is convex on $\left[r_{1}, r_{2}\right]$ for $q \geq 1$, then the following inequality holds:

$$
\begin{gathered}
|E(f, Q)| \leq \frac{1}{32 \sqrt[q]{3}} \times \sum_{i=0}^{k-1}\left(x_{i+1}-x_{i}\right)^{2} \\
\times\left\{\sqrt[q]{2\left|f^{\prime}\left(\frac{3 x_{i}+x_{i+1}}{4}\right)\right|^{q}+\left|f^{\prime}\left(x_{i}\right)\right|^{q}}\right. \\
+\sqrt[q]{2\left|f^{\prime}\left(\frac{x_{i}+x_{i+1}}{2}\right)\right|^{q}+\left|f^{\prime}\left(\frac{3 x_{i}+x_{i+1}}{4}\right)\right|^{q}} \\
+\sqrt[q]{2\left|f^{\prime}\left(\frac{x_{i}+3 x_{i+1}}{4}\right)\right|^{q}+\left|f^{\prime}\left(\frac{x_{i}+x_{i+1}}{2}\right)\right|^{q}} \\
\left.+\sqrt[q]{\left|f^{\prime}\left(\frac{x_{i}+3 x_{i+1}}{4}\right)\right|^{q}+2\left|f^{\prime}\left(x_{i+1}\right)\right|^{q}}\right\} .
\end{gathered}
$$

Proof. The proof is analogous as to that of Proposition 3.6 but use Theorem 2.12 . 
Remark 3.8. Applying our Theorems 2.3 and 2.12 for special value $m=1$ and various suitable choices of function $\varphi(t)=\frac{t^{\alpha}}{\Gamma(\alpha)}, \frac{t^{\frac{\alpha}{k}}}{k \Gamma_{k}(\alpha)}, \varphi(t)=t\left(r_{2}-\right.$ $t)^{\alpha-1}$ and $\varphi(t)=\frac{t}{\alpha} \exp \left[\left(-\frac{1-\alpha}{\alpha}\right) t\right]$ for $\alpha \in(0,1)$, such that $\left|f^{\prime}\right|^{q}$ to be $m$ convex, we can deduce some new bounds for the trapezium formula using above ideas and techniques. The details are left to the interested reader.

\section{Acknowledgements}

The author would like to thank the referee for valuable comments and suggestions.

\section{REFERENCES}

[1] S.M. Aslani, M.R. Delavar and S.M., Vaezpour, Inequalities of Fejér type related to generalized convex functions with applications, Int. J. Anal. Appl., 16(1) (2018), $38-49$.

[2] F.X. Chen and S.H. Wu, Several complementary inequalities to inequalities of Hermite-Hadamard type for s-convex functions, J. Nonlinear Sci. Appl., 9(2) (2016), $705-716$.

[3] Y.M. Chu, M.A. Khan, T.U. Khan and T. Ali, Generalizations of Hermite-Hadamard type inequalities for MT-convex functions, J. Nonlinear Sci. Appl., 9(5) (2016), 43054316.

[4] M.R. Delavar and M. De La Sen, Some generalizations of Hermite-Hadamard type inequalities, SpringerPlus, 5(1661) (2016).

[5] S.S. Dragomir and R.P. Agarwal, Two inequalities for differentiable mappings and applications to special means of real numbers and trapezoidal formula, Appl. Math. Lett., 11(5) (1998), 91-95.

[6] G. Farid and A.U. Rehman, Generalizations of some integral inequalities for fractional integrals, Ann. Math. Sil., 31 (2017), pp. 14.

[7] A. Kashuri and R. Liko, Hermite-Hadamard type fractional integral inequalities for generalized $(r ; s, m, \varphi)$-preinvex functions, Eur. J. Pure Appl. Math., 10(3) (2017), 495-505.

[8] A. Kashuri and R. Liko, Hermite-Hadamard type inequalities for generalized $(s, m, \varphi)$ preinvex functions via $k$-fractional integrals, Tbil. Math. J., 10(4) (2017), 73-82.

[9] A. Kashuri and R. Liko, Some new Hermite-Hadamard type inequalities and their applications, Stud. Sci. Math. Hung., 56(1) (2019), 103-142.

[10] M.A. Khan, Y.M. Chu, A. Kashuri and R. Liko, Hermite-Hadamard type fractional integral inequalities for $M T_{(r ; g, m, \varphi)}$-preinvex functions, J. Comput. Anal. Appl., 26(8) (2019), 1487-1503.

[11] M.A. Khan, Y.M. Chu, A. Kashuri, R. Liko and G. Ali, New Hermite-Hadamard inequalities for conformable fractional integrals, J. Funct. Spaces, (2018), Article ID 6928130, pp. 9.

[12] T. Lara, On strongly m-convex functions, Math. Aeterna, 5(3) (2015), 521-535.

[13] W. Liu, W. Wen and J. Park, Hermite-Hadamard type inequalities for MT-convex functions via classical integrals and fractional integrals, J. Nonlinear Sci. Appl., 9 (2016), 766-777. 
[14] C. Luo, T.S. Du, M.A. Khan, A. Kashuri and Y. Shen, Some $k$-fractional integrals inequalities through generalized $\lambda_{\phi m}-M T$-preinvexity, J. Comput. Anal. Appl., 27(4) (2019), 690-705.

[15] M.V. Mihai, Some Hermite-Hadamard type inequalities via Riemann-Liouville fractional calculus, Tamkang J. Math, 44(4) (2013), 411-416.

[16] S. Mubeen and G.M. Habibullah, k-Fractional integrals and applications, Int. J. Contemp. Math. Sci., 7 (2012), 89-94.

[17] O. Omotoyinbo and A. Mogbodemu, Some new Hermite-Hadamard integral inequalities for convex functions, Int. J. Sci. Innovation Tech., 1(1) (2014), 1-12.

[18] M.E. Özdemir, S.S. Dragomir and C. Yildiz, The Hadamard's inequality for convex function via fractional integrals, Acta Mathematica Scientia, 33(5) (2013), 153-164.

[19] M.Z. Sarikaya and F. Ertuğral, On the generalized Hermite-Hadamard inequalities, https://www.researchgate.net/publication/321760443.

[20] M.Z. Sarikaya and H. Yildirim, On generalization of the Riesz potential, Indian Jour. of Math. and Mathematical Sci., 3(2), (2007), 231-235.

[21] E. Set, M.A. Noor, M.U. Awan and A. Gözpinar, Generalized Hermite-Hadamard type inequalities involving fractional integral operators, J. Inequal. Appl., 169 (2017), $1-10$.

[22] H. Wang, T.S. Du and Y. Zhang, k-fractional integral trapezium-like inequalities through $(h, m)$-convex and $(\alpha, m)$-convex mappings, J. Inequal. Appl., 2017(311) (2017), pp. 20.

[23] X.M. Zhang, Y.M. Chu and X.H. Zhang, The Hermite-Hadamard type inequality of GA-convex functions and its applications, J. Inequal. Appl., (2010), Article ID 507560, pp. 11.

[24] Y. Zhang, T.S. Du, H. Wang, Y.J. Shen and A. Kashuri, Extensions of different type parameterized inequalities for generalized $(m, h)$-preinvex mappings via $k$-fractional integrals, J. Inequal. Appl., 2018(49) (2018), pp. 30.

Artion Kashuri

Department of Mathematics,

Faculty of Technical Science, University Ismail Qemali, Vlora, Albania

E-mail address: artionkashuri@gmail.com 